\title{
Preface
}

In my dissertation (Dik 1968) I criticised the theory of Transformational Grammar in the form which it had at that time (the so-called 'Standard Theory' of Chomsky 1965) for its treatment of coordinative constructions. I argued that for certain types of coordination a transformational description is syntactically and semantically inadequate, and that for other types such a description is not necessary inasmuch as non-transformational grammars can be devised which can express the required distinctions. I ascribed the fact that Phrase structure grammars of the format incorporated into Transformational Grammars cannot express the required distinctions, to the circumstance that such grammars, while specifying the categorial structure of linguistic expressions, do not make explicit the grammatical functions of constituents, so that categorially identical but functionally different constituents cannot be distinguished, and categorially distinct but functionally equivalent constituents cannot be identified, except in roundabout and counterintuitive ways. As an alternative to Transformational Grammar I sketched a model of 'Functional Grammar', in which such grammatical functions as Subject and object figured among the primitives of grammatical description, and I demonstrated that different types of coordination could be handled in terms of this model, given certain principles of assigning grammatical functions to constituents.

In the meantime linguistic theory has seen many developments. Some of these have made it clear to me that my 1968 sketch of a Functional Grammar was rather too primitive to attain descriptive and explanatory adequacy in the description of natural languages. Other developments have shown, however, that the basic idea underlying this sketch, namely the adoption of functional notions as primitives of grammatical theory, is a fruitful one which has so far been insufficiently explored.

Thus, Fillmore (1968) demonstrated the relevance of semantic functions in grammatical description. Keenan (1972 etc.) showed that non-trivial cross-linguistic generalisations can be formulated in terms of ₹unctional notions. Ideas of Perlmutter and Postal generated a number of studies in 'Relational Grammar', in which grammatical functions appear as theoretical primitives. Finally, different theoreticians, most notably the members of the 'Prague School', have pointed out the relevance of what we shall term 'pragmatic functions'.

In this book I develop a theory of Functional Grammar with the following main distinguishing properties: (i) it is conceived from a functional point of view on the nature of language: 
that is, one in which a language is regarded as an instrument of social interaction; (ii) it makes crucial use of functional notions of three different levels: the semantic, the syntactic, and the pragmatic level; (iii) it starts with the construction of abstract underlying predications on the basis of predicate-frames contained in the lexicon; (iv) these underlying predications are mapped onto linguistic expressions by means of expression rules which specify the form and the order in which constituents with given functional and categorial properties can or must appear; (v) it avoids the use of transformations in the sense of structure-changing operations.

Many people have helped me to give this book its present form. In a general way I should like to acknowledge the inspiration which I have derived from numerous discussions with students and colleagues about different aspects of Functional Grammar.

I am very grateful to Connie Menting for typing assistance and general support, to Patricia Muysken and Norval Smith for correcting my English, to Irene Cannegieter for expertly typing the final text, and to willy van wetter for general encouragement and understanding.

Holysloot, September 1977

SIMON C. DIK

\section{PREFACE TO THE THIRD PRINTING}

This third printing of Functional Grammar, which enjoys the hospitality of a new publisher, is identical to the first, except for the following points:

(a) a number of minor errors, mostly typos, have been corrected. I am grateful to those who pointed these out to me.

(i) Figure 1 on 0.23 replaces the earlier, less perspicuous schema.

(c) chapter 10 now gives a very brief outline of further work in Functional Grammar, and some references to this work. 